\title{
LE CERNEST : \\ Centre de référence \\ neuromusculaire \\ Grand Est
}

\author{
Andoni Echaniz-Laguna ${ }^{1}$, Christine Tranchant ${ }^{1}$, \\ Vincent Laugel ${ }^{2}$, Cyril Schweitzer ${ }^{3}$, \\ Maud Michaud ${ }^{4}$, François Boyer ${ }^{5}$, Pascal Sabouraud ${ }^{6}$
}

Le Centre de Référence des Maladies Neuromusculaires Rares de la région Grand-Est (CERNEST) est un centre multi-sites labellisé en 2006. II rassemble les équipes médicales et les compétences scientifiques des CHU de Strasbourg, Nancy et Reims. Ses objectifs principaux sont la prise en charge des patients et la recherche clinique et fondamentale. L'enseignement aux professionnels de santé et la diffusion de l'information médicale au grand public fait également partie des missions du CERNEST sur son territoire.

Sur chacun des trois sites, des consultations spécialisées destinées à la prise en charge des enfants et des adultes ont été développées. Le Centre couvre l'ensemble des maladies neuromusculaires incluant les myopathies, les maladies du système nerveux périphérique, les myasthénies et les amyotrophies spinales à l'exclusion de la SLA (centres de référence spécifiques à Strasbourg et Nancy). Plus de 3000 patients sont reçus chaque année au CERNEST. IIs sont suivis en consultations simples ou pluridisciplinaires et si nécessaire en hospitalisation classique ou en hôpital de jour. En fonction des besoins, les patients peuvent consulter sur chaque site des neurologues, pédiatres, médecins rééducateurs, cardiologues, pneumologues ou généticiens. Des équipes paramédicales regroupant infirmières, diététiciennes, psychologues, ergothérapeutes, kinésithérapeutes et assistantes sociales sont également disponibles sur chaque site. Des laboratoires spécialisés assurent les explorations neuromusculaires - EMG, biopsie musculaire, biopsie de nerf - mais également les analyses histologiques et les tests génétiques.

\section{Le CERNEST : un centre de référence intégré au sein de la nouvelle région Grand Est}

La labellisation du CERNEST en 2006 a structuré les équipes et organisé la prise en charge des patients

au sein de la nouvelle région Grand Est. De multiples efforts ont été consacrés à l'harmonisation des

\author{
${ }^{1}$ Département de Neurologie, \\ CHU Hautepierre, \\ Strasbourg, France. \\ ${ }^{2}$ Département de Pédiatrie, \\ CHU Hautepierre, \\ Strasbourg, France. \\ ${ }^{3}$ Département de Pédiatrie, \\ Hôpital d'Enfants - CHU de \\ Brabois, Nancy, France. \\ ${ }^{4}$ Département de Neurologie, \\ Hôpital Central, Nancy, France. \\ ${ }^{5}$ Service de médecine physique \\ et de réadaptation, CHU de Reims, \\ France. \\ ${ }^{6}$ Département de Pédiatrie, \\ CHU de Reims, France. \\ andoni.echaniz-laguna@ \\ chru-strasbourg.fr
}

modes de fonctionnement par la mise au point de procédures communes. Les liens avec l'AFM-Téléthon, qui soutient financièrement les consultations spécialisées, ont été formalisés et les directeurs des services régionaux AFM participent régulièrement aux réunions du CERNEST. Enfin, le centre joue un rôle actif dans la Filière Nationale Neuromusculaire (FILNEMUS).

\section{Une activité de recherche clinique et fondamentale de premier plan}

La recherche est une activité prioritaire du CERNEST, comme l'attestent les nombreuses publications scientifiques issues de notre centre depuis 2006. Plusieurs membres du CERNEST appartiennent à des équipes de recherche Inserm, CNRS ou universitaires tant à Strasbourg qu'à Nancy et Reims. Ils consacrent une part significative de leur activité à la recherche clinique et fondamentale, notamment par le biais d'un travail en réseau avec les Centres d'Investigations Cliniques. Des projets de recherche thérapeutiques régionaux ont ainsi pu voir le jour, comme par exemple le Projet Hospitalier de Recherche Clinique (PHRC) UPACOMT (Ulipristal Acétate dans la maladie de Charcot-Marie-Tooth de type $1 A$ - A. Echaniz-Laguna, Strasbourg) qui fédère l'ensemble des équipes du CERNEST dans un projet commun, ou le projet «Qualité de vie et maladies neuromusculaires » (F. Boyer, Reims). 

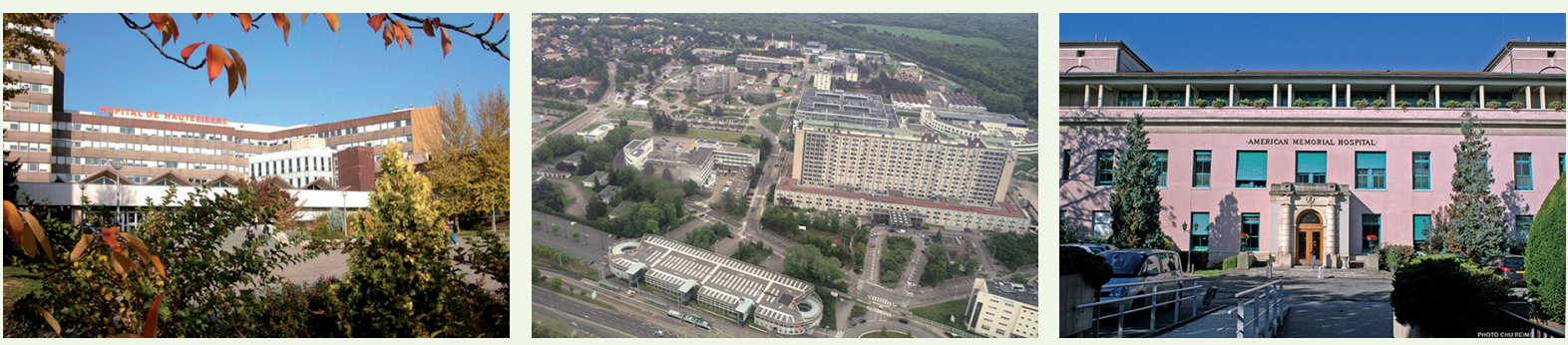

La région Grand Est abrite par ailleurs de nombreuses équipes de LIENS D'INTÉRÊT recherche Inserm et CNRS qui participent à la vie du CERNEST par la mise au point de nouvelles techniques de séquençage génétique, indispenLes auteurs déclarent n'avoir aucun lien d'intérêt concernant les données publiées dans cet article sables au diagnostic complexe des maladies neuromusculaires (Projet Myocapture dont l'objectif est la recherche de nouveaux gènes responsables de myopathie, en collaboration avec le laboratoire Généthon et I'Institut de Myologie, J. Laporte et V. Biancalana, Strasbourg). $\diamond$

\section{Bulletin d'adhésion 2016}

NOM/Prénom :
$\square$ Clinique
$\square$ Fondamentale
$\square$ Autre fonction

Adresse :

Code Postal : Ville :

E-mail :

ADHÉSION : Je désire adhérer en qualité de (rayer la mention inutile)

Membre titulaire : $40 €$

Membre étudiant : gratuit (fournir un justificatif de votre qualité d'étudiant non salarié)

RÈGLEMENT

$\square$ Je joins un chèque libellé à l'ordre de la Société Française de Myologie d'un montant de $40 €$

$\square$ J'effectue un virement bancaire de $40 €$

A RETOURNER A :

Rémi.mounier@univ-lyon1.fr

ou

Rémi MOUNIER - Trésorier de la SFM

CR HDR CNRS - UMR CNRS 5534

Centre de Génétique et de Physiologie Moléculaire et Cellulaire

Université Claude Bernard Lyon 1

Bâtiment Gregor Mendel - 2è étage

16 Rue Raphaël Dubois

F-69622 Villeurbanne Cedex

\section{N.B. : Bulletin à photocopier et à diffuser à toute personne intéressée}

